\title{
Culturad Par \\ ¿CALIDAD DE LA EDUCACIÓN \\ O NUEVO PARADIGMA DE EDUCACIÓN?
}

Recibido: 20.08.2014 / Aprobado: 16.09.2014

Francisco José Lacayo Parajón ${ }^{1}$

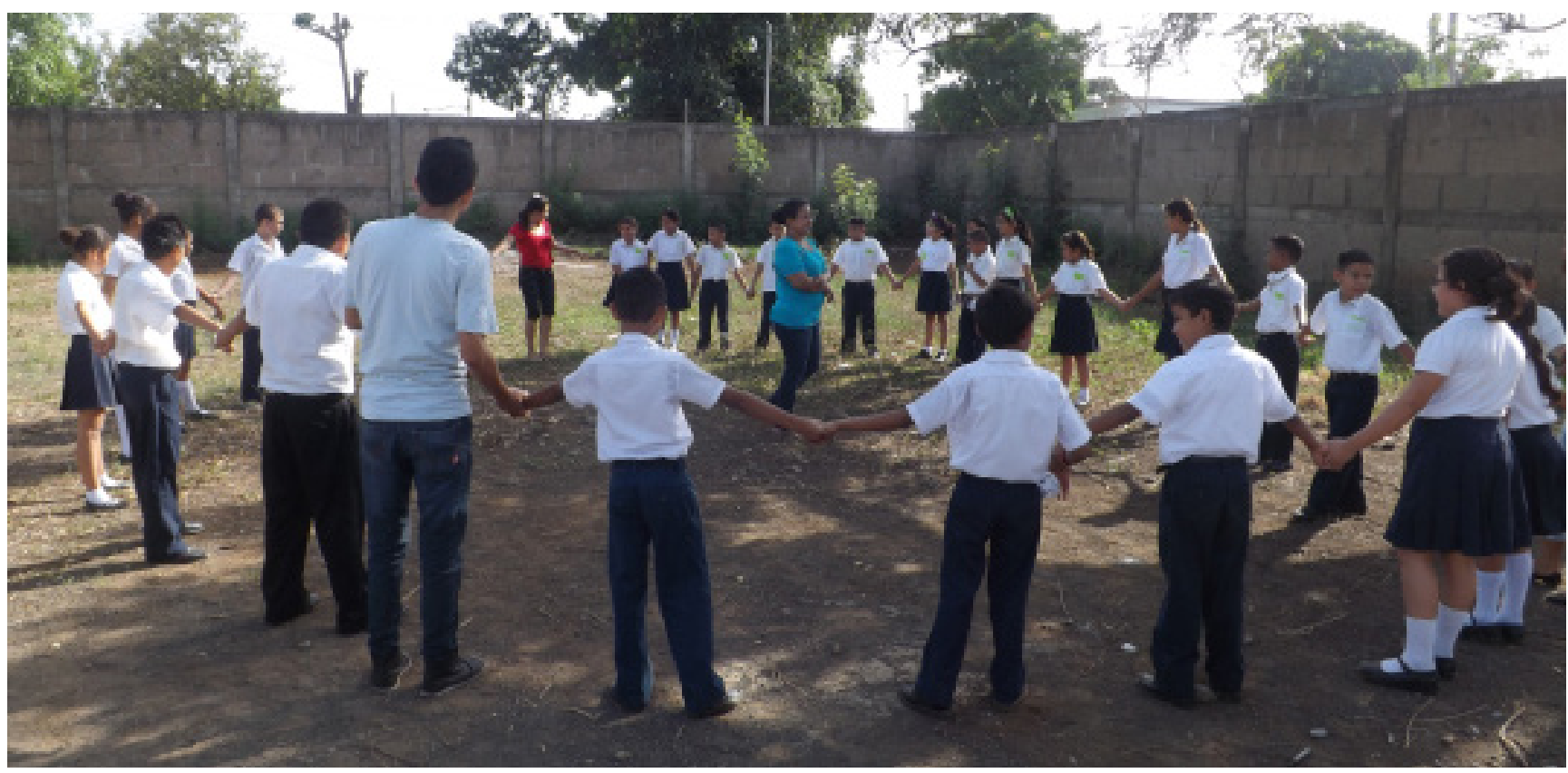

Foto: Soren Chamorro

\section{RESUMEN}

Francisco Lacayo Parajón se centra en analizar estructural y holísticamente el reto que el paradigma de educación nos plantea en el siglo XXI, no sólo a nosotros sino al mundo entero. En su opinión la educación está en el corazón de la paz y de la cultura de paz. El Acta Constitutiva de la UNESCO afirma que para evitar una nueva guerra hay que erigir la paz a través de la educación, la ciencia y la cultura.

Palabras clave: Educación, calidad, reinvención, paradigma, desarrollo.

\section{ABSTRACT}

Abstract: Francisco Lacayo Parajón focuses on analyzing structural and holistically the challenge that the education paradigm considers in the 21st century, not only to us but the whole world. In his opinion, education is at the heart of peace and of a culture of peace. The Constitutive Act of the UNESCO states that to avoid a new war one must build peace through education, science, and culture.

Key Words: education, quality, reinvention, paradigm, development. 


\section{Hasta el cambio ha cambiado}

En todas las épocas ha habido cambios pero, en la época actual, puede decirse que hasta el concepto de cambio ha cambiado.

En las últimas décadas, los más grandes paradigmas de la humanidad han entrado en procesos de reinvención que exigen definir nuevos contratos entre la sociedad y esos paradigmas.

En el famoso documento de la UNESCO Nuestra Diversidad Creativa, los estados miembros de la Organización nos dicen:

“ El desafío que tiene ante sí la humanidad es adoptar nuevas formas de pensar, actuar y organizarse en sociedad; en resumen nuevas formas de vivir. El desafío consiste también en promover vías de desarrollo diferentes, informadas por el reconocimiento de cómo los factores culturales modelan la manera cómo las sociedades conciben sus propios futuros y eligen los medios para alcanzarlos."

"Este momento realmente extraordinario de la historia requiere soluciones de excepción. El mundo que conocemos, todas las relaciones que dábamos por sentadas están experimentando una reformulación y una reconstrucción profundas. Se

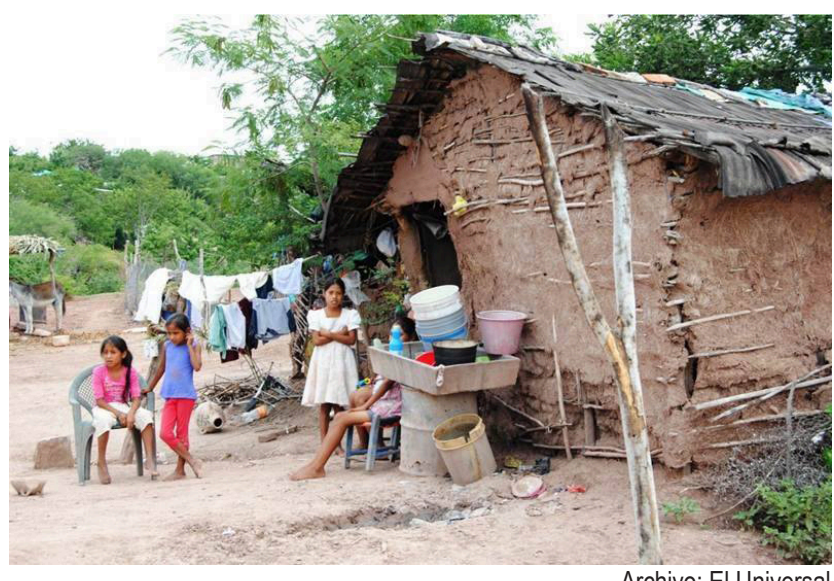

necesita imaginación, capacidad de innovación, visión y creatividad." 2

Permítanme reflexionar brevemente sobre el cambio de algunos de los grandes paradigmas de la humanidad en las últimas décadas, entre ellos el paradigma de educación.

\section{La reinvención del paradigma ambiental. La Madre Tierra}

La Conferencia Internacional de Naciones Unidas sobre el Medio Humano (conocida posteriormente como Cumbre de la Tierra de Estocolmo), convocada por Naciones Unidas en 1972, fue la primera gran conferencia de la ONU sobre cuestiones ambientales internacionales. En ella el mundo asumió el paradigma de desarrollo sostenible.

Veinte años después, en la Cumbre de la Tierra de Río de Janeiro (1992), convocada también por Naciones Unidas, 108 jefes de estado y de gobierno redefinieron el contrato de la sociedad con la Madre Tierra, mal llamada recurso natural o medio ambiente.

Desde la Cumbre de la Tierra, en Río de Janeiro en 1992, el viejo concepto de medio ambiente o recursos naturales ha seguido evolucionando, hasta el nuevo paradigma de la Madre Tierra, Pacha Mama, la Tonantzin de Nicaragua, que, a propuesta de los pueblos andinos de Nuestra América, fue asumido por la Asamblea General de Naciones Unidas, decretando, el 22 de abril de cada año, día internacional de la Madre Tierra. ${ }^{3}$

"En este nuevo paradigma la Tierra y todos los seres de la naturaleza, particularmente los vivos y los animales son titulares de derechos. Estos deben ser incluidos en las constituciones modernas que han abandonado el arraigado antropocentrismo y el paradigma del dominus, del ser humano como señor y dominador de la naturaleza y de la Tierra."4

2 Nuestra Diversidad Creativa. Informe de la Comisión Mundial de Cultura y Desarrollo. UNESCO. 1996

3 Resolución aprobada por la Asamblea General el 22 de abril de 2009. Resolución 63/278. Día Internacional de la Madre Tierra [sin remisión previa a una Comisión Principal (A/63/L.69 y Add.1)] 63/278. Día Internacional de la Madre Tierra

4 "... un notable criminalista y juez de la Corte Suprema de la Argentina, Eugenio Raúl Zaffaroni (La Pachamama y el Humano, Ediciones Colihue 2012) desarrolla un pensamiento constitucionalista de naturaleza ecológica en el cual la Tierra y todos los seres de la naturaleza, particularmente los vivos y los animales son titulares de derechos. Estos deben ser incluidos en las constituciones modernas que han abandonado el arraigado antropocentrismo y el paradigma del dominus, del ser humano como señor y dominador de la naturaleza y de la Tierra". Leonardo Boff. Teólogo/Filósofo. Constitucionalismo ecológico en América Latina. 14 mayo 2013 
$\mathrm{Al}$ argumentar en defensa del paradigma Madre Tierra-Pachamama, Ollanta y Itzamná afirma: "la Pachamama (Madre Tierra) ...no es sólo natura o physis. Mucho menos res extensa o un reloj mecánico. Ella no es una materia insensible o irracional. La Tierra, con todos los seres que la cohabitamos, es nuestra única Madre Tierra. Tiene la mayor dignidad y los derechos mayores. Por ello la respetamos y la amamos como hijos e hijas. De Ella venimos, en Ella convivimos, y hacia Ella retornamos. Nuestros derechos, dignidad, autoconsciencia y espiritualidades dependen de los derechos, la dignidad, la autoconsciencia y la espiritualidad de Ella." ${ }^{15}$

\section{La necesaria reinvención del fracasado modelo de desarrollo}

Incluso el fracasado paradigma de desarrollo, eje de la actual sociedad de mercado, intenta remendarse desesperadamente, aunque no ha surgido todavía, con vigor suficiente, un nuevo paradigma alternativo.

El binomio desarrollo-subdesarrollo, presentado como paradigma globalizante de la humanidad, nació en 1947, a propuesta del Presidente de los Estados Unidos de Norteamérica, Harry Truman, quien lo presentó al mundo en sustitución del nefasto paradigma "civilización y barbarie".

Este aspirante a paradigma, eje de la actual sociedad de mercado, se presentó como un sustantivo, no siendo más que un falaz adjetivo y, para camuflar su ineficacia en más de seis décadas, ha tenido que inventarse, cada vez más, nuevos adjetivos: "desarrollo sustentable", "desarrollo con equidad social" etc. y, el más triste y falaz de todos, el "desarrollo humano", en donde lo humano es relegado al estatus de adjetivo.

La crisis mundial que estalló en 2008, y que impactó en el corazón del fracasado modelo de desarrollo, sigue vigente y los teóricos del capitalismo no logran superar, seis años después, su total desconcierto.

La realidad es que el reto actual ya no estriba en diseñar e implementar un desarrollo sustentable, sino una sustentabilidad de la vida, amenazada de extinción acelerada por el viejo modelo de desarrollo.

Incluso la bien intencionada propuesta de desarrollo sustentable, se ha vuelto insuficiente. El reto real ahora es la sustentabilidad de la vida. Sólo después podrá definirse el modelo social y económico que sea pertinente.

En la actual sociedad de mercado, todos los paradigmas que forman parte de la cosmovisión vigente de la humanidad son tributarios de este concepto agotado e inviable de desarrollo.

No obstante, amerita señalar que también, en este campo, viene surgiendo, desde Nuestra América, una propuesta alternativa: el Bien Vivir, contrapuesto al Vivir Mejor consumista e inviable.

\section{La crisis actual es integral y múltiple}

La crisis actual es integral y no se limita, ni es, en primer lugar, a como sostienen Wall Street y el G8, una crisis financiera o monetaria, sino que ella es también y en primer lugar, una crisis energética, una crisis alimentaria, una crisis de recursos hídricos y desertificación y la más grave de todas, una crisis de irreversible cambio climático.

Todas estas crisis son hijas de la obcecación de un sistema económico, regido por los dogmas de continuo crecimiento y la maximización sin límites de las ganancias.

En un reciente seminario, en abril 2013, organizado por el Fondo Monetario Internacional para repensar la política económica mundial, el premio Nobel de Economía George Akerlof, describió con una metáfora su total incertidumbre: "Es como si un gato se hubiera subido a un árbol enorme. El gato, por supuesto, es la crisis.-dice- Mi posición es 'Dios mío, ese gato se va a caer y no sé qué hacer!'

David Romer, intervino después y dijo: "El gato ha estado en el árbol por cinco años. Es hora de obligarlo a bajar y asegurarse de que no se vuelva a subir" (sic).

Otro de los participantes, el premio Nobel Joseph Stiglitz, sostuvo que el problema estriba en que "no hay una buena teoría económica que explique por qué el gato todavía está en el árbol". Eso parecía un foro de veterinarios.

El economista en jefe del FMI,Oliver Blanchard, cerró la conferencia diciendo: "No hemos identificado nuestro destino final a dónde llegaremos, realmente no tengo ni idea". Esto amerita el premio nóbel a la sinceridad. ${ }^{6}$ Poco después, en mayo del mismo año, el Papa Francisco, señalaba la dimensión ética que olvidaron

5 Radio Habana Cuba. Boletín Pedro Martínez Pirezpmpirez@rhc.cu 18 de junio 2014

6 http://www.bbc.co.uk/mundo/noticias/2013/04/130422 economia crisis sin solucion finde.shtml

http://www.am.com.mx/opinion/lagosdemoreno/no-sabemos-que-hacer-george-akerlof-1497.html 
los expertos en economía y denunciaba el sistema financiero global que ...explota a los pobres y transforma a los seres humanos en bienes de consumo descartables" "El origen último -de la crisis financiera- dijo, está en una profunda crisis humana".

\section{El cambio cualitativo en el paradigma de la cultura}

Si queremos abordar el reto por una educación con calidad que, a mi entender, es una educación coherente, pertinente $\mathbf{y}$ eficaz, es ineludible reflexionar, al mismo tiempo, sobre el cambio del paradigma de cultura, en las últimas décadas.

El viejo paradigma de cultura que ha regido hasta ahora, vive también, desde hace cuatro décadas, una transformación profunda $\mathrm{y}$, en principio, positiva, expresada en tratados internacionales, consensuados por casi todos los estados del mundo. En el caso de Nicaragua, estos grandes consensos han sido ratificados por la Asamblea Nacional Legislativa y tienen carácter vinculante. Entre otras muchas, menciono la Convención para la Salvaguardia del Patrimonio Cultural Inmaterial (París, octubre, 2003) y la Convención sobre la Protección y Promoción de la Diversidad de las Expresiones Culturales (París, octubre, 2005).

El viejo concepto de cultura que ha regido hasta ahora está fuertemente marcado, por la tesis de "civilización o barbarie", con la que los poderes esclavistas y colonizadores definieron, impusieron y legitimaron un concepto que puede resumirse así: son civilizados (cultos) los conquistadores triunfantes y son bárbaros (incultos) los pueblos conquistados o sometidos.

Desde esa lógica, la milenaria cultura China y la ingeniería y arquitectura de las pirámides mayas, no fueron más que expresiones "bárbaras" e "incultas".

Manipulando avances científicos, como la teoría de Darwin, se elaboraron los falsos conceptos dogmáticos de raza, raza superior, pueblos sin cultura, pueblos incultos, cultura culta, entre otros.

Hay que afirmarlo con una sana y legítima intransigencia: no hay buen salvaje, ni salvaje malo, no hay barbaros, ni civilizados, no hay razas ni culturas superiores, sólo hay diversidad de riquezas, complementarias $y$, en aquellas hasta ahora subvaloradas, se esconden muchos de los secretos que pueden abrir el camino para la salvación de la humanidad y de todas las expresiones de la vida, en nuestro único hogar, la Madre Tierra, si todavía eso es posible.

En la Conferencia Mundial contra el Racismo, la Discriminación Racial, la Xenofobia y las Formas Conexas de Intolerancia (Durban, Sudáfrica, 2001) los estados del mundo calificaron los conceptos y prácticas de esclavitudes, colonizaciones y discriminaciones racistas como "crímenes contra la humanidad" y rechazaron "enérgicamente toda doctrina basada en la superioridad racial, así como las teorías que pretenden demostrar la existencia de razas humanas presuntamente distintas..."

Tras la segunda guerra mundial, Harry Truman, propuso la substitución de la dicotomía "civilización y barbarie" por la de "países desarrollados-países subdesarrollados". El resto del mundo de la postguerra asumió sin controversias esta tesis.

Pero, en esta propuesta, continuaron camuflados y vigentes los conceptos de "alta cultura", "pueblos incultos", "culturas superiores".

Paradójicamente, al menos una buena parte de los mismos sectores que entronizaron el concepto de civilización y barbarie, se han convertido, en las últimas décadas, en los promotores de un nuevo paradigma, que trata de recuperar la dimensión humanista y salvífica de la cultura, en era de la globalización.

Este movimiento abre a nuestros pueblos del Sur, una ventana para las propuestas creativas, siempre y cuando pasemos antes por un proceso de descolonización cultural. El siglo XXI es el momento de las culturas emergentes con propuestas salvíficas para toda la humanidad.

Claro está que esta opción pide un proceso de desaprendizaje, de eso que Freud llamaría "policías internos", y que Bolívar califica de "dolencias de servidumbre", interiorizados e institucionalizados por siglos, en nuestras mentes y costumbres. Para asumir el nuevo paradigma de cultura, tenemos que conocer y re-conocer las raíces de nuestra personalidad histórica material e inmaterial -siempre mestiza-, recrearlas, nacionalizarlas y universalizarlas.

Esto ya sucedió en la historia de nuestra Nicaragua. El español no es una lengua autóctona de los pueblos precolombinos. El mestizo Rubén Darío injertó en ella, como una yema, nuestras raíces milenarias, la enriqueció de otras diversidades universales (clásicas y modernas), la re-creó y la devolvió totalmente renovada a los pueblos hermanos en donde tuvo su origen. 
Además de ser reconocido como Héroe Cultural de Nicaragua, Renovador Universal de la lengua española, Príncipe de las letras castellanas, "el ciudadano más cabal e ilustre de América Latina", al decir de Pedro Salinas, Rubén es una de las raíces patrimoniales más determinantes de la nicaragüanidad. Aún más, Darío es el Gran Libertador Cultural de Nuestra América. Así lo reconoce el gran escritor Jorge Luis Borge al afirmar: “...quienes alguna vez lo combatimos, lo llamamos Libertador."

En 1821 nuestros pueblos centroamericanos firmaron el Acta de la Independencia política, territorial y administrativa. Nos hace falta redactar e implementar el Acta de la Independencia Cultural.

Pero, antes de injertar en nuestra identidad cultural lo más excelente de las diversidades universales, es preciso discernir, re-conocer, valorizar y articular las raíces y el potencial de nuestra personalidad histórica y cultural, ricamente mestiza. Es preciso nacionalizar y universalizar todos los rostros de nuestra nicaragüanidad. A partir de allí, todo lo que es humano nos pertenece y podemos, imitando a Darío, injertarlo y renovarlo.

No olvidemos que, hasta 1980, se desconocía oficialmente la existencia de uno de los rostros excelentes de nuestra personalidad histórica: la cultura garífuna, reconocida por la UNESCO, en 2003, como Patrimonio de la Humanidad.

Los múltiples rostros de la nicaragüanidad no se limitan al Pacífico o al Güegüence. Hay rostros de nuestra identidad en el Caribe, en los pueblos del norte y del centro de Nicaragua. La opción ante nuestra rica diversidad no pasa por un proceso de integración, sino por la sabia articulación equitativa de esas diversidades.

\section{¿Qué no es la cultura?}

Quiero señalar brevemente algunos de los falsos y desfasados conceptos de cultura que, aunque descartados en los consensos de los tratados internacionales de las últimas décadas, siguen vigentes en muchas mentes políticas y académicas, como las "dolencias de servidumbre" de las que nos habla Simón Bolívar.

1. Cultura no es sinónimo de educación escolar moderna, la cual no tiene más de quinientos años de existencia. Los diplomas académicos no son los indicadores de nuestra cultura, sino una parte de ella.

2. Cultura no es sinónimo de modernidad. La identificación de cultura con modernidad ha descartado como incultas y atrasadas, por ejemplo, las sabidurías milenarias sobre la salud humana, que no son deudoras de la joven medicina moderna, ni de la poderosa industria farmacéutica.

3. La Cultura no es solamente pilar de identidad, ella es también pilar de soberanía y factor determinante para el desarrollo humano, social y económico, así como garantía de una globalización proactiva, con soberanía e identidad. Hay pueblos que han sobrevivido sin territorio, ninguno ha sobrevivido sin identidad cultural.

4. La Cultura no se limita a las "bellas artes", definidas en el pasado por los poderes dominantes. Esas "bellas artes" son cultura pero, ni abarcan la totalidad, ni son per se el prototipo universal de la excelencia. Cada diversidad cultural puede y debe definir sus propios paradigmas de belleza y de excelencia. "Nuestro vino de plátano, y si es agrio es nuestro vino" dijo Martí.

Para esos viejos conceptos, nietos de la falacia de "civilización y barbarie", resulta imposible reconocer con la UNESCO la excelencia cultural universal de nuestro Güegüence y de la Lengua, Danza y Música del pueblo Garífuna, expresiones ambas declaradas Patrimonio Cultural Inmaterial de la Humanidad.

5. Los recursos asignados a la cultura no son una "carga" o un gasto para la sociedad o el estado. Todo recurso asignado al mundo de la cultura es una inversión para el desarrollo humano, social y económico, si esta asignación es coherente con el nuevo paradigma de cultura.

\section{Los tres grandes espacios operativos de la cultura: Patrimonio, diversidad y creatividad}

En el nuevo paradigma de cultura se pueden discernir tres grandes dimensiones que trascienden el viejo concepto de cultura: la diversidad cultural, la creatividad de las sociedades e individuos y el patrimonio cultural (como un fenómeno raíz-viva y no como una momia suntuaria) 
La relación entre estas tres dimensiones de la cultura es dialéctica e indisoluble. El patrimonio es fruto de la creatividad/diversidad y a su vez alimenta la renovación permanente de esa creatividad y de esa diversidad.

La diversidad cultural es una condición de la creatividad. Crear es engendrar algo diverso desde la propia identidad. Esta creatividad será más rica y viva en la medida en la que se alimente de sus raíces patrimoniales, que son una forma institucionalizada de la diversidad (como es el caso de las lenguas).

Desde hace tres décadas vengo proponiendo una definición operativa y metafórica, ${ }^{7}$ de la cultura como "la personalidad histórica, material e inmaterial de una sociedad", inspirada en las ciencias sicológicas y sociales.

En la salvaguarda y re-creación de las riquezas de patrimonio, diversidad y creatividad, juega un papel estratégico la educación, sea esta formal, no formal o informal. No hay que olvidar que la culturización es la primera y más importante modalidad educativa. Elementos tan importantes como la educación en valores escapa a la jurisdicción de la "escuela formal" y su promoción sólo se logra a través de la "culturización".

Al igual que personalidades individuales, las culturas poseen varias ascendencias o paternidades y desde sus raíces históricas van definiendo y afirmando la diversidad de su identidad y, por lo tanto, su potencial de creatividad.

Las personalidades culturales, al igual que las de los individuos, son procesos y no actos puntuales, que evolucionan con mayor o menor sanidad, según sea la capacidad de enfrentar los embates internos o externos, positivos o negativos de su historia y de su contexto geográfico y ecológico.

\section{La riquezas culturales motor de desarrollo}

Todas estas visiones falsas y desfasadas ignoran que, en las últimas décadas, la cultura ha sido reconocida por la casi totalidad de los estados miembros de la UNESCO y de otros organismos de Naciones Unidas, como un factor ineludible del desarrollo humano, social e incluso económico, ya que su contribución permea y marca todas las actividades de la vida.

Quisiera enfatizar que no puede haber una reinvención del paradigma de educación, sin una sabia articulación con el nuevo paradigma de cultura y desarrollo.

Es desde esta nueva concepción que, hace pocos años, el documento Estrategia de Cultura y Desarrollo, de la Cooperación Española, afirma: "Quizás el gran esfuerzo que se reclama es la necesidad de una mayor concreción de las potencialidades de la cultura como motor de desarrollo..." Y añade: "La cultura se define, no ya como una dimensión accesoria del desarrollo, ni como un elemento de nostalgia histórica o solamente de identidad sino como el tejido mismo de la sociedad y como fuerza interna para su desarrollo."

Ya en 1977, el Informe Mundial de Cultura y Desarrollo: "Nuestra Diversidad Creativa", leemos: "El papel de la cultura no se reduce a ser un medio para alcanzar fines, sino que constituye la base social de los fines mismos. El desarrollo y la economía forman parte de la cultura de los pueblos".

En la Mesa Redonda de Ministros de la Cultura del Mundo, (París, diciembre 2000) convocada por la UNESCO, los participantes afirman: "La cultura no debe ocupar una plaza secundaria ante la economía" "Las industrias culturales son las industrias del futuro..."

\section{¿Calidad de la educación o un nuevo paradigma de educación?}

¿Qué educación y para qué modelo de desarrollo humano, social y económico?

Actualmente se reflexiona mucho sobre la calidad de la educación pero, no es posible definir los criterios de la calidad en la educación, si antes no se define qué entendemos por educación y qué se espera de ella para los individuos y en la sociedad.

Con frecuencia se repite como eslogan que "sin educación no hay desarrollo". Pero, ¿de qué educación y de qué desarrollo estamos hablando?

La terrible tragedia de la segunda guerra mundial tuvo como actores principales a los países que en esa época habían alcanzado el mayor nivel en educación escolar moderna y el mayor nivel en las ciencias y las tecnologías. Ese modelo de educación,

7 La primera definición de cultura, conocida en occidente, utiliza también una metáfora. Esta definición se la se la debemos a Cicerón, quien inspirándose en la metáfora de la agricultura, acuñó el término de cultura aplicándolo a la sociedad. 
de desarrollo y de ciencia no impidió la masacre más grande conocida en la historia, con más de 40 millones de muertos y el inicio guerrerista de la energía nuclear para exterminar poblaciones enteras.

Si cualquier modelo de educación garantizara un desarrollo humanista, portador de los derechos humanos, de la convivencia pacífica, de la equidad social -a nivel nacional y a nivel internacional-, del reconocimiento y valoración de la diversidades y del rechazo a todo tipo de discriminación, marginación, esclavismo, racismo y opresión, no habría habido segunda guerra mundial, ni hubieran sido creadas Naciones Unidas y la UNESCO, organización que surge tras la segunda guerra mundial afirmando, en su Acta Constitutiva, "Ya que la guerra nace en la mente de los hombres, es en la mente de los hombres donde hay que erigir los baluartes de la paz".

\section{¿Qué es la calidad en la educación? Un vistazo a la tierra, desde la luna}

Para reflexionar en el siglo XXI sobre la educación, tenemos que hacer el ejercicio de "mirar la tierra desde la luna". Es decir, salirnos por un momento de la cápsula del modelo educativo escolar vigente y reflexionar sobre cómo implementaron otras sociedades sus sistemas educativos y cómo definieron la calidad de la educación.

Toda sociedad humana ha tenido un sistema educativo, cualquiera que haya sido su modalidad. Actualmente la hegemonía la tiene el sistema educativo escolar, vigente desde hace menos de 500 años. No obstante muchas reflexiones actuales sobre la educación y su calidad, se plantean como si el actual modelo de educación escolar fuera natural e inherente a las sociedades humanas y el único vigente y posible en el siglo XXI.

¿Podemos seguir ignorando que la institución escolar formal se achica cada vez más en sus pretendidas funciones, ante el desarrollo de los procesos de información y educación no escolar que han implementado fenómenos como Windows, Facebook, Google y similares Estas son verdaderas "escuelas" que cuentan con miles de millones de "estudiantes" voluntarios, de todas las edades, creencias, lenguas y condiciones sociales?

$\mathrm{Su}$ impacto es tan profundo que trasciende el campo de la "enseñanza-aprendizaje" y está globalizando una nueva cultura, un nuevo lenguaje, una nueva formación y desarrollo de la personalidad de su "alumnado-clientela", unas nuevas formas de relación social, particularmente en la niñez y la juventud.

Las masas ingentes de comunicación e información, acompañadas de nuevas modalidades de interrelación social, asumen la transformación de todos los espacios de la educación humana: valores, actitudes, modelos de comportamiento, conocimientos, destrezas y habilidades.

Así mismo estas modalidades de "informacióncomunicación-formación" se han convertido en uno de los campos más rentables y codiciados de la sociedad de mercado en todos sus espacios.

Quienes realmente han asumido y están alcanzando la propuesta de la UNESCO de construir una sociedad educativa, son esas empresas mundiales de información y comunicación y no el sistema escolar oficial. Lamentablemente esa globalización educativa se está haciendo, no desde la visión unesquiana, sino desde la motivación del lucro empresarial de la sociedad de mercado. Más adelante aportaremos unas modestas reflexiones sobre el ¿ qué hacer? ante estos cambios profundos.

Esta "visión de la tierra desde la luna" nos permite percibir quela definición de la calidad de una educación está condicionada por el modelo de sociedad que queremos reproducir y hacer evolucionar.

Impuestas por la sociedad de mercado, en el mundo moderno dos mitologías se erigen como políticas de la educación para la sociedad de consumo: el empleo y el éxito. Desgraciada la sociedad que acepta únicamente estas metas como punto de partida, para definir la calidad educativa.

Ante el evidente fracaso del modelo actual de la sociedad de mercado, donde el termómetro del éxito en la educación y, por tanto, de la calidad educativa, está determinado por su aporte a la ganancia sin límites del 1\% de la población, detentora del $40 \%$ de las riquezas de la humanidad, se impone el reto de la búsqueda de "un mundo posible diferente", de un paradigma alternativo de sociedad.

La invención de un modelo alternativo de sociedad conlleva la invención de un modelo alternativo de educación.

Lamentablemente, hoy en día, la mayoría de las propuestas alternativas para la educación, están lastradas por la "dolencia de servidumbre" de la que nos hablaba Simón Bolívar. 
Por lo tanto, el reto de reinventar un modelo educativo adecuado está en relación simbiótica con la reinvención del modelo de sociedad y de desarrollo y a su vez, es un factor determinante para el nuevo modelo de sociedad.

¿Qué educación, para qué modelo de sociedad, para qué modelo de persona humana en el siglo XXI, tenemos que inventar en la Nicaragua de hoy y del mañana próximo?

\section{¿Cómo construyeron en el pasado las sociedades humanas sus modelos educativos?}

Cada sociedad en la historia definió primero su modelo de sociedad y su modelo ideal de la persona humana y, en función de ellos, como objetivos y resultados, definió su modelo educativo.

Primero -aunque no en prioridad temporal- se define el modelo de sociedad y de persona humana a la que la sociedad aspira y luego se define e implementa un modelo educativo (formal, no formal, informal, comunitario) que supuestamente garantice alcanzar esas metas de sociedad y de persona humana.

En las sociedades que nos precedieron, los sistemas educativos se identificaban casi totalmente con los procesos de socialización comunitaria y culturización.

El modelo era "la sociedad educativa". Educar era reproducir y hacer evolucionar en forma coherente y eficaz la personalidad histórica de una sociedad, es decir, su identidad en todas las dimensiones.

Esta identidad surge:

a) del modelo de relación considerada pertinente entre la sociedad y la naturaleza.

b) del modelo de relación con otras sociedades extranjeras, c del modelo de cosmovisión propio de cada sociedad y de su estatus y función en el contexto concreto.

Esta "visión de la tierra desde la luna" nos permite percibir que la definición de la calidad de una educación está condicionada por el modelo de sociedad que queremos reproducir $y$ hacer evolucionar.

\section{La educación encierra un tesoro}

En los foros sobre educación se afirma el consenso universal acuñado en la UNESCO de que las metas para la educación en siglo XXI deben de ser:

a) cobertura universal de la educación y

b) educación con calidad.

Estas tesis son ciertas y válidas pero, insuficientes como bases para la acción. Se mantiene la pregunta ¿de qué educación estamos hablando?

En 1996, la UNESCO promovió un informe trascendental sobre este tema: Informe a la UNESCO de la Comisión Internacional sobre la Educación para el Siglo XXI. La comisión de sabios la presidió Jacques Delors, exministro francés y el documento es conocido como "La educación encierra un tesoro".

En ese documento la Unesco plantea para la educación del siglo XXI los siguientes pilares: ${ }^{8}$

Aprender a ser, aprender a hacer, aprender a aprender, aprender a convivir. ${ }^{9}$

Muchos de sus planteamientos siguen siendo válidos pero, casi dos décadas después, es necesario actualizarlos.

Así por ejemplo, Federico Mayor Zaragoza, ex Director General de la UNESCO incorporó otro pilar: "aprender a emprender".

8 El realce en negrillas es del autor. fi

9 - "Aprender a conocer, combinando una cultura general suficientemente amplia con la posibilidad de profundizar los conocimientos en un pequeño número de materias. Lo que supone además: aprender a aprender para poder aprovechar las posibilidades que ofrece la educación a lo largo de la vida.

- Aprender a hacer a fin de adquirir no sólo una calificación profesional sino, más generalmente, una competencia que capacite al individuo para hacer frente a gran número de situaciones y a trabajar en equipo.

Pero, también, aprender a hacer en el marco de las distintas experiencias sociales o de trabajo que se ofrecen a los jóvenes y adolescentes, bien espontáneamente a causa del contexto social o nacional, bien formalmente gracias al desarrollo de la enseñanza por alternancia.

- Aprender a vivir juntos desarrollando la comprensión del otro, el reconocimiento y valoración de las diversidades y la percepción de las formas de interdependencia -realizar proyectos comunes y prepararse para tratar los conflictos- respetando los valores de pluralismo, comprensión mutua y paz.

- Aprender a ser para que florezca mejor la propia personalidad y se esté en condiciones de obrar con creciente capacidad de autonomía, de juicio y de responsabilidad personal. Con tal fin, no menospreciar en la educación ninguna de las posibilidades de cada individuo: memoria, razonamiento, sentido estético, capacidades físicas, aptitud para comunicar.

- Mientras los sistemas educativos formales propenden a dar prioridad a la adquisición de conocimientos, en detrimento de otras formas de aprendizaje, importa concebir la educación como un todo. En esa concepción deben buscar inspiración y orientación las reformas educativas, tanto en la elaboración de los programas como en la definición de las nuevas políticas pedagógicas." Informe a la UNESCO de la Comisión Internacional sobre la Educación para el Siglo XXI. Segunda Parte Capítulo 4. Pistas y recomendaciones. 1996 
En mis reflexiones sobre la educación, a lo largo de estos años, he sugerido incluir el "aprender a desaprender" y en el apartado de "aprender a convivir" propongo extender este principio más allá de la sociedad humana e incluir el "aprender a convivir con la Madre Tierra".

Así mismo considero que aprender a crear, aprender a innovar, desde la propia identidad, son también pilares de la educación para este siglo, quizás como una explicitación necesaria del "aprender a hacer" de la Comisión.

Permítanme desglosar algunas reflexiones sobre estos pilares de la educación y proponer algunos otros, frutos de las reflexiones sobre esta temática.

Educación para convivir. El documento de la UNESCO focaliza esta línea en el campo de la convivencia social y fundamentalmente orientada hacia la razón de ser de la Organización: la paz. Actualmente tendríamos que incorporar también la convivencia de la sociedad humana con la madre tierra, como un ser vivo, y no como un insumo, ni siquiera como un medio ambiente o como un entorno natural, que la humanidad puede explotar a su gusto y antojo,. La madre tierra es un ser vivo al que pertenecemos las personas humanas. Nosotros necesitamos más de ella que ella de nosotros $\mathrm{y}$, hasta ahora, tras milenios de irracional explotación es necesario educar para una relación con la madre tierra marcada por la equidad y el intercambio vital entre ambas partes. El prestigiado científico Edward Wilson afirma que si la humanidad desapareciera de pronto, los insectos no tendrían ninguna preocupación pero, si ellos desaparecen nuestra humanidad no puede seguir existiendo.

Amerita señalar aquí que la paz como razón de ser de la UNESCO, alcanzó un mayor nivel de explicitación y desarrollo en esta organización, en los años 90', cuando bajo la brillante conducción del Director General de la UNESCO, en ese entonces, Don Federico Mayor Zaragoza propuso al mundo no la paz en general sino la cultura de paz, única garantía de una paz firme, duradera y base del "Bien Vivir".

Educar para aprender a aprender. Es preciso que la educación del siglo XXI asuma concretamente la tesis de que, en el binomio enseñanza -aprendizaje, lo más importante es el aprendizaje. Asimismo es ineludible priorizar la formación de aptitudes y actitudes sobre el almacenamiento de conocimientos. Ante el estallido violento de masas de información, que desbordan cualquier capacidad intelectual humana y cualquier desarrollo de la memoria, los diferentes niveles educativos han cesado de tener un punto terminal. A diferencia de siglos pasados, actualmente, en cuanto una persona termina de graduarse en cualquier nivel de la educación formal escolar, se encuentra ya en un estado de "analfabetismo", ante la masa ingente de nuevos conocimientos que han surgido en ese período.

Asimismo, aunque esta tesis no se encuentra en el mencionado documento de la UNESCO, es preciso dar una importancia estratégica a la educación sobre las tendencias actuales que nos permiten vislumbrar desde ahora las posibles opciones de la sociedad humana en un futuro relativamente cercano, que de alguna forma está naciendo ya en el presente. Es preciso más que nunca, una educación proyectada al futuro, cada vez más inmediato, a causa del ritmo acelerado de las grandes crisis que la especie humana enfrenta, en los inicios del siglo XXI, tales como el cambio climático, la crisis alimentaria, la crisis energética, la crisis ecológica en general, y la crisis financiera y monetaria y la globalización dominante de las nuevas tecnologías de comunicación e información.

Aprender a desaprender, en primer lugar los viejos, fracasados o superados paradigmas, a partir de la comprensión de los nuevos paradigmas de la humanidad. Este reto es muy exigente. Einstein afirmaba que es más fácil desintegrar un átomo que un prejuicio. Lo primero que hay que desaprender es la aceptación fetichista y cuasi sagrada de los viejos paradigmas. Es el momento de los "por qué" de la escuela socrática. Es el momento del desarrollo máximo de la educación crítica, orientada hacia una actitud propositiva.

Educar para aprender a emprender a partir de la creatividad. Una educación centrada en el desarrollo de las aptitudes, en el desarrollo de la facultad de aprender a aprender, en el conocimiento análisis y comprensión de las tendencias que definen desde ahora las posibles opciones del futuro a muy corto plazo, necesariamente tiene que conllevar, en forma sistemática, la formación de las personas en la creatividad y en el emprendimiento.

Aprender a reconocer y valorar las diversidades. Es preciso reconocer lo que afirma la Unesco "la diversidad cultural es tan importante para la especie humana como la biodiversidad para los seres vivos". 
No hay sociedad sustentable posible (supuesto fin del desarrollo sustentable) sin el conocimiento, re-conocimiento, valorización de las diversidades como insoslayables y como complementariamente enriquecedoras.

Aprender a dialogar. Nadie nace sabiendo dialogar, como nadie nace sabiendo hablar, leer o escribir. El diálogo en la educación pasa por el proceso del reconocimiento positivo de las diversidades, vistas como riqueza y no como amenaza. Una globalización sin el crecimiento axial del diálogo entre las diversidades no puede engendrar más que un dominio totalitario en muy pocas manos.

Aprender a negociar. Es necesario educar en la negociación no a pesar y contra las diversidades sino a partir de ellas.

No hay opción de sobrevivencia para la especie humana si no es a partir de una negociación, que si no es sustentada por valores éticos o morales debe ser motivada, al menos, por un pragmatismo de sobrevivencia. La negociación no es monopolio de la política o de los negocios. En todas nuestras experiencias humanas estamos siempre negociando espacios diversos del "yo", del "tú", del "nosotros" del "ellos".

Aprender a concertar. Considero que la concertación es un nivel superior a la negociación. Tomando del campo de las artes musicales la figura de concierto, hay que educar en la asociación de proyectos de "bien común". En la música, los diferentes instrumentos solistas, sin renunciar a su identidad y a su diversidad propias, puedan articularse en función de crear un nuevo producto musical, que ninguno de ellos podría crear por sí solo: el concierto. La diferencia entre el ruido y una sinfonía de Bach es la concertación.

Aprender a desarrollar la inteligencia emocional y espiritual

Me limito, por razones de espacio a citar aquí unas reflexiones de ese gran pensador e intelectual que es Leonardo Boff: "Toda la modernidad se ha construido casi exclusivamente sobre la inteligencia intelectual. Ella nos ha traído innumerables comodidades. Pero no nos ha hecho más integrados y felices porque ha puesto en segundo plano y hasta ha reprimido la inteligencia emocional y cordial, y ha negado la ciudadanía a la inteligencia espiritual. Hoy es urgente amalgamar estas tres expresiones de la inteligencia si queremos desentrañar esos valores y sentimientos en los que tiene su nicho: el respeto, la reverencia y la coexistencia pacífica con la naturaleza y la Tierra. Este enfoque nos alinea con la lógica de la naturaleza que se consorcio, interretro-conecta a todos y con todos y sostiene la sutil red de la vida.". ${ }^{1011}$

\section{Educar para la creatividad como pilar de la calidad educativa}

Permítanme una reflexión especial sobre la educación para la creatividad, en el campo de la calidad educativa.

No hay sociedad ni cultura sin creación, más aún: la cultura es creatividad o no es cultura.

10 Boff, Leonardo. "El Papa Francisco, ¿promotor de la conciencia ecológica?” Columnases@voila.fr. 14 abril 2013.

11 Boff, Leonardo "Entre las muchas formas de inteligencia estudiadas hoy, destacan tres en particular: la inteligencia intelectual, la inteligencia emocional y la inteligencia espiritual. Todas ellas basadas en serias investigaciones científicas.

Por medio de la inteligencia intelectual (el famoso $\mathrm{Cl}$ ) organizamos nuestros pensamientos, articulamos los diversos saberes, en especial el lenguaje y estrategias de la acción. Está ligada a la dimensión del cerebro llamada neocortex. Este es relativamente nuevo en el proceso de la antropogénesis. No tiene más de siete u ocho millones de años, pero se desarrolló completamente con la aparición del homo sapiens sapiens hace unos cien mil años. La obra civilizadora con sus artes y ciencias se deriva del neocortex. Sin él no entenderíamos el mundo de hoy, no sobreviviríamos. Pero la inteligencia intelectual sola no da cuenta de la vida humana.

Existe en nosotros la inteligencia emocional, estudiada particularmente por Daniel Goleman en su famoso libro Inteligencia Emocional (1995). Está relacionada con el cerebro límbico que surgió hace más de 130 millones de años cuando en la evolución aparecieron los mamíferos. Estos llevan la cría dentro de sí y, una vez nacida, la rodean de amor y cariño. Surgió entonces en el universo conocido algo absolutamente nuevo: la emoción, el afecto, el sentimiento, la pasión, el amor y también sus contrarios. Nosotros, los humanos, olvidamos que somos mamíferos intelectuales y racionales. Las capas más profundas y decisivas de nuestra vida tienen esta historia antigua. Somos ante todo seres de emoción y de sentimiento.

Goleman demostró que la primera reacción humana frente a cualquier fenómeno no es intelectual/racional, sino emocional. Unos momentos después entra la inteligencia racional/intelectual. Algunos filósofos (Meffesoli,Cortina, Scheler, MunizSodré, Duarte Jr.) la llaman también "razón cordial, o sensible». Goleman critica la inflación de la inteligencia racional que ha vuelto a las personas competitivas, individualistas e insensibles y dadas a la violencia en lugar de ser más solidarias, compasivas y humanitaria. Sugiere una verdadera «alfabetización emocional» a partir de la escuela, algo que he estado postulando desde hace 20 años con los libros Saber cuidar y El cuidado necesario. Él disminuye la violencia en todos los campos. La inteligencia emocional es el nicho de los valores, de la ética, del amor, y lo que da sentido a nuestras vidas Finalmente existe en nosotros la inteligencia espiritual. Durante los últimos veinte años ha habido un fuerte desarrollo de la neurociencia, la neurolingüística y otras afines que estudian el cerebro humano. En él hay miles y miles de millones de neuronas y trillones de sinapsis (conexiones entre ellas). Se hizo un descubrimiento sorprendente: cada vez que una persona se ocupa existencialmente con visiones globales de las cosas, con el sentido de la vida, con lo sagrado y con Dios, en su lóbulo frontal se da una aceleración inusitada de las neuronas. Danah Zohar, científica cuántica, con su marido psiquiatra lan Marshall han resumido sus muchas búsquedas en un libro titulado Inteligencia espiritual (2000). " en artículo "Todavía mística y espiritualidad para futbolistas" Columnases@voila.fr. 14 abril 2013. Remitido por L. Boff por correo electrónico 
Durante siglos se arrinconó a la creatividad en el espacio de las bellas artes, cuando ella es el útero, el crisol en el que una cultura renace continuamente en todas sus dimensiones. La creatividad es uno de los rasgos distintivos de la especie humana. La meta de la calidad educativa exige la promoción de la creatividad en todos los campos. La ciencia, las artes, la economía, la resolución de problemas sobre todo los más graves no existen sin una promoción máxima de la creatividad

"Crear es la palabra de pase" repetía José Martí.

La creatividad es uno de los rasgos distintivos de la especie humana. La meta de la calidad educativa exige la promoción de la creatividad en todos los campos, tanto en la ciencia como en las artes, la economía, la resolución de problemas, la organización social, etc. Los desafíos que enfrenta y enfrentará cada vez más la humanidad en el siglo XXI no podrán resolverse correctamente sin una verdadera "cultura de la creatividad".

La sociedad de mercado ha comprendido, desde su visión e intereses esta necesidad. En el campo de la economía se ha acuñado ya el concepto de industrias creativas, cada vez más importantes en el mundo moderno. En este apartado se ubican también las llamadas "industrias protegidas por derechos de autor"

"Las industrias creativas pueden definirse como los ciclos de creación, producción y distribución de bienes y servicios que utilizan creatividad y capital intelectual, como insumos primarios ellas comprenden un conjunto de actividades basadas en el conocimiento que producen bienes y servicios intelectuales o artísticos al inglés y de contenido creativo, valor económico y un colectivo de mercado". ${ }^{12}$

A manera de ejemplo, según el economista Ernesto Piedras, las industrias protegidas por los derechos de autor (PDA) de Méjico, aportaron el 7\% al PIB del país. (Piedras, 2004).

Uno de los campos que la creatividad está redefiniendo con más protagonismo y fuerza es el mundo de la informática, las comunicaciones, la web.

Por otra parte hay que señalar que la creatividad es un proceso y no un suceso aislado y prodigioso de generación espontánea.

A veces se sigue pensando en ella como el chispazo mágico pero, "la creatividad y la capacidad de formular nuevas ideas y su implementación en la generación de productos o servicios ordenando de una manera diferente a la existente de la materia y el trabajo humano, podemos ver que no hay nada más alejado que el mágico y chispazo...". ${ }^{13}$

Los grandes creadores, tanto en el campo de las llamadas bellas artes. como en la ciencia, en la técnica en la organización social, en la comunicación etc., están hermanados en la dimensión universal humana de la creatividad.

Pero, para crear nuevas alternativas en necesario romper los viejos moldes. La educación con calidad debe promover, como proceso, la formación en la creatividad y por lo tanto, personalidades en actitud creativa, capaces de romper los viejos y fracasados moldes y paradigmas del status quo.

La creatividad debe de ser coherente, pertinente y eficaz con los retos que enfrenta la humanidad. Más de una vez, la creatividad consiste en recuperar sabidurías ancestrales y eficaces, desechadas por la sociedad de mercado por no considerarlas "mercancías suficientemente rentables". Las ciencias médicas son un campo en el que esta forma de creatividad puede desarrollarse más.

Pero, la formación de personalidades creativas en cualquier proceso educativo, debe articularse dialécticamente con el conocimiento, reconocimiento y re-creación de las raíces patrimoniales y de sus riquezas de diversidad.

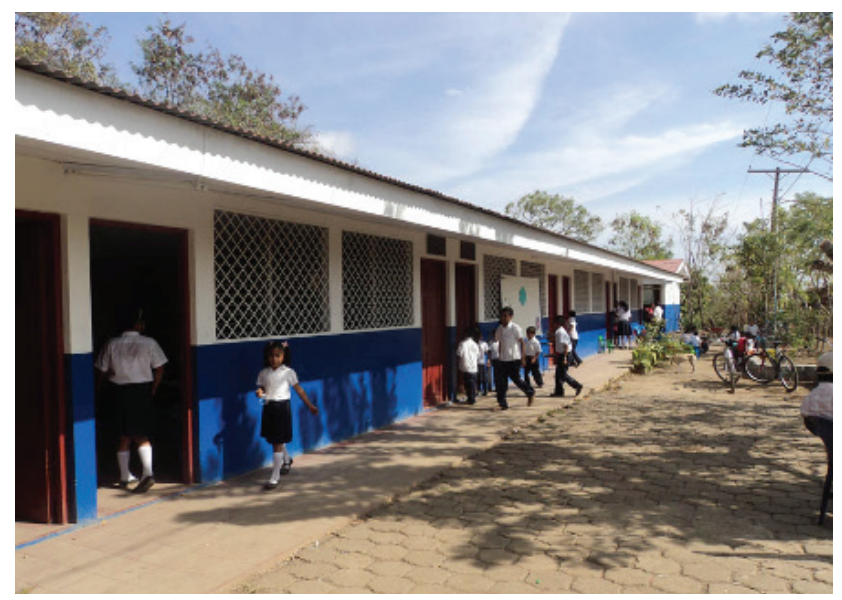

www.bolsadenoticias.com.ni

12 Cfr.Informe sobre la economía creativa 2008 Naciones Unidas 2008. p. 63.

13 Economía y cultura. Coordinadores: Marisa Reyes Godínez, Jorge Linares Ortiz. Editorial Universidad autónoma de la Ciudad de México, México septiembre 2003. "El valor de la creatividad: algunas reflexiones en busca de método." Hugo Álvarez Ravelo.Pág. 117 y ss. 


\section{¿Calidad de la educación o éxito en el sistema, en beneficio de los que se lucran de él?}

El actual concepto de educación ha sido monopolizado por una economía de mercado cuya verdadera globalización consiste en convertir a toda la sociedad en una sociedad de mercado, sin dejar ningún espacio que se escape a la definición, función y estatus de mercancía.

Es a partir de una crítica acompañada de una propuesta alternativa al falso y fracasado paradigma de desarrollo económico social y humano que podemos sentar las bases de un nuevo paradigma de educación.

El reto de definir desde un nuevo paradigma de educación la dimensión de la calidad educativa es tanto más grande cuanto que esa tarea debe emprenderse en contradicción con una cosmovisión y un sistema hegemónicos de sociedad y desarrollo que se resiste a esta innovación.

La sociedad de mercado insiste en definir la educación como un "instrumento" para alcanzar el desarrollo, entendiendo por esto fundamentalmente el crecimiento económico de una sociedad, lo que hasta ahora nunca ha sido equitativo. Con esa concepción reduce a las personas a la categoría de "capital humano" dentro de un supuesto paradigma de desarrollo cuya intencionalidad cada vez más descarada es convertir todo, incluso el mundo de la cultura, de lo simbólico, de la identidad, de lo espiritual en mercancía.

Esta visión imperante entra en abierta contradicción con uno de los consensos más positivos, aunque imperfecto e inacabado.de la Carta de los Derechos Humanos, aprobada por la totalidad de los estados del mundo, en donde la educación es definida en primer lugar como un derecho.

Estas dos visiones están en profunda contradicción.

La lógica es que los derechos humanos no necesitan demostrar en primer lugar, que son "instrumentos útiles". Aunque, hoy más que nunca, ante la profunda crisis de valores salvíficos de la vida, es válida la frase de José Martí, en su prólogo a Ismaelillo "Creo... en la utilidad de la virtud.."

El derecho a la vida, por ejemplo, no toma su valor y legitimidad por alguna contribución al desarrollo, cualquiera que sea la connotación de este concepto

$\mathrm{Al}$ igual que Windows, con sus permanentes "parches", el falaz concepto hegemónico de desarrollo se ha visto obligado a adjuntarse un calificativo más en su larga lista de "parches": el desarrollo humano. Pero, como más arriba señalamos, la palabra desarrollo sigue manteniendo el estatus de sustantivo y asigna a la persona humana el estatus de adjetivo.

Es, desde el escenario de estas contradicciones, que debemos de ir construyendo nuevos parámetros para la calidad educativa.

En el mundo moderno dos mitologías se erigen como políticas axiales de la educación para la economía de mercado, convertida en sociedad de mercado: el empleo y el éxito.

Desgraciada la sociedad que acepta únicamente estas metas como punto de partida, para definir la calidad educativa.

Ante el evidente fracaso del modelo actual de la sociedad de mercado, donde el termómetro del éxito en la educación y, por tanto, de la calidad, está determinado por el aporte del $99 \%$ de la humanidad a la ganancia sin límites del $1 \%$ de la población, detentora del $40 \%$ de las riquezas de la humanidad, se impone el reto de la búsqueda de "un mundo posible diferente", de un paradigma alternativo de sociedad.

Este modelo alternativo de sociedad, conlleva la invención de un modelo alternativo de educación pertinente, viable, coherente y eficaz para la creación de la sociedad sustentable.

¿Qué educación, para qué modelo de sociedad, para qué modelo de persona humana en el siglo XXI, tenemos que inventar en la Nicaragua de hoy y del mañana próximo?

\section{Los criterios de la calidad educativa}

Antes y por encima de pensum, currícula, métodos, instrumentos didácticos etc. la calidad educativa en la historia de la humanidad se ha guiado y se debería seguirse guiando por unos parámetros fundamentales.

En estas cortas reflexiones me permito plantear algunos de esos criterios.

1. La educación debe de ser coherente:

a) Con los nuevos paradigmas, alternativos a los viejos paradigmas fracasados, con la especificidad y contexto de la sociedad involucrada, con su cultura y sus recursos. 
b) Con el plan de desarrollo nacional, siempre y cuando éste trascienda los límites de servidumbre que le impone la sociedad de mercado y que se plantee en una articulación indisoluble entre desarrollo humano desarrollo social y desarrollo económico.

c) Con la búsqueda de una sociedad basada en la satisfacción de las necesidades y no en un consumo sin límites alimentado permanentemente por la creación de nuevas "necesidades", que impone en forma globalizada la sociedad de mercado.

d) Con la identidad y el contexto de la propia nación, de la propia comunidad pero, asimismo coherente con la identidad, siempre diversa de la humanidad en todas sus expresiones regionales nacionales o comunitarias.

2. La educación debe de ser pertinente con el aquí y el ahora (hic et nunc). Pero, en el siglo XXI, el "ahora" es también el mañana inmediato. A diario estamos discerniendo tendencias de la sociedad que nos permiten vislumbrar el futuro mediato. La educación tiene que ser pertinente para el hoy y para el mañana próximo y cuasi presente, no sólo a nivel universal sino, en primer lugar a nivel nacional y local.

Es necesario un proceso educativo que asuma, entre otras, las graves advertencias que nos hace llegar el eminente biólogo Edward O Wilson, uno de los cien científicos más prestigiados del mundo actualy creador del concepto biodiversidad en el mundo científico. Wilson nos informa que, de continuar el deterioro actual de la naturaleza, en el año 2030 es inminente la desaparición de cientos de miles de millones de toneladas del hielo marino estival en el Ártico, con todas las catastróficas consecuencias que eso supone, entre ellas la liberación de inmensas masas de gas metano. ${ }^{13}$

¿Cómo podríamos plantear una propuesta educativa pertinente, coherente y eficaz, ignorando hoy ese tipo de tendencias?

Así mismo, una educación con calidad debe de incluir la formación en el reconocimiento a la diversidad y a la solidaridad de y entre las sociedades.

El mundo político y académico, aunque cita con frecuencia la frase que sintetiza la razón de ser de la UNESCO, tras la horrenda pesadilla de la segunda guerra mundial: “...puesto que las guerras nacen en la mente de los hombres, es en la mente de los hombres donde deben erigirse los baluartes de la paz"; parecen haber olvidado otros textos estratégicos del Acta Constitutiva de la UNESCO: “...una paz fundada exclusivamente en acuerdos políticos y económicos entre gobiernos no podría obtener el apoyo unánime, sincero y perdurable de los pueblos, y que, por consiguiente, esa paz debe basarse en la solidaridad intelectual y moral de la humanidad."

Es evidente la contradicción entre una propuesta educativa orientada al "éxito, a través de la competitividad egoísta" y una educación basada en la solidaridad intelectual y moral de la humanidad.

Así mismo se ocultan o ignoran artículos fundamentales de esa misma Acta, redactada por "Los gobiernos de los Estados Partes en la presente Constitución, en nombre de sus pueblos" tales como: "Que, en el curso de la historia, la incomprensión mutua de los pueblos ha sido motivo de desconfianza y recelo entre las naciones, y causa de que sus desacuerdos hayan degenerado en guerra con harta frecuencia; "

"Que la grande y terrible guerra que acaba de terminar no hubiera sido posible sin la negación de los principios democráticos de la dignidad, la igualdad y el respeto mutuo de los hombres, y sin la voluntad de sustituir tales principios, explotando los prejuicios y la ignorancia, por el dogma de la desigualdad de los hombres y de las razas..."

"Que la amplia difusión de la cultura y la educación de la humanidad para la justicia, la libertad y la paz son indispensables a la dignidad del hombre y constituyen un deber sagrado que todas las naciones han de cumplir con un espíritu de responsabilidad y de ayuda mutua;"

Este documento histórico no fue redactado por monjes o líderes espirituales, sino por los más humanistas, sensatos y realistas de los políticos sobrevivientes de la gran pesadilla de la segunda guerra mundial.

\section{La educación debe de ser eficaz}

Pero, esta eficacia debe de estar en función de la persona humana, de la vida en todas sus expresiones y no del mercado. La humanidad necesita una

13 Científicos del Grupo de Emergencia del Metano en el Ártico (AMEG) aseguran que la masiva liberación del metano 'encerrado' en la masa de hielo del Ártico a la atmósfera constituye una situación de "emergencia planetaria". El efecto invernadero del gas metano es veinte veces más potente que el dióxido de carbono. por los efectos negativos de esta liberación sobre el calentamiento global. 
educación eficaz para sobrevivir, para "bien vivir" y para detener el suicidio de la humanidad y el ecocidio exterminador de la vida. La eficacia en la calidad educativa se encuentra en relación simbiótica con la coherencia y la pertinencia de la educación.

Las "especializaciones" crecientes en los procesos educativos están en función de la eficacia del imperio de la sociedad de mercado, no de las necesidades materiales, sociales, emocionales y espirituales de las personas humanas. El crecimiento y los intereses del 1\% de la sociedad humana no garantiza ni podrá garantizar nunca el "bien vivir" del 99\% restante.

Esta eficacia debe de superar la falacia de los criterios de éxito que impone la sociedad de mercado.

¿Y cuál es este éxito para los poderes imperantes en la sociedad de mercado?

En el mes de junio 2013, el famoso presentador de CNN Ismael Cala fue invitado por sectores empresariales y medios de comunicación a exponer a los nicaragüenses el camino del éxito. ${ }^{14}$

Según Cala, en la vida podemos contar la historia como víctimas o como gladiadores y aclaró que estos últimos a lo mejor no son los que tienen la victoria sino los que no se vencen (sic). En su caso particular, nos dice que prefiere hacerlo como gladiador, a pesar de haber estado rodeado de pobreza y limitaciones.

Completando su receta para el "éxito" el presentador de CNN añade:" Ríete de la vida, hay que desconfiar del que no tiene sentido del humor", "Enfoca tu mente en la gratitud: sé feliz con lo que tienes y no pienses en lo que te hace falta", "Crea tu propia filosofía y escríbela" ?15 ¿Qué pensarán de esta receta mágica los miles de hombres y mujeres nicaragüenses con graduación universitaria, que no han logrado conseguir empleo digno y los más de cien mil nicaragüenses quequedarán sin empleo en los cortes de café a consecuencia de la roya y el cambio climático?

Como este recetario, hay miles que son divulgados en libros bestseller y por todos los medios de comunicación. Si Cala y la revista Vanidades respondieran a nuestros grandes interrogantes en educación, no tendríamos que estar perdiendo el tiempo en tantos foros sobre calidad educativa.

Una de las impertinencias de Cala y compañía, (literalmente es una falta de pertinencia) es que dichas recetas se presentan como "universales" y desconocen totalmente la diversidad de condiciones de las personas humanas.

Lamentablemente, Cala fue invitado con honores y centenares de personas acudieron al joven gurú, buscando en sus palabras y en su experiencia el mágico secreto del éxito.

El criterio de la eficacia para el bien vivir, nos lleva por su propia lógica al campo de los contenidos educativos.

En primer lugar, como hemos afirmado más arriba, al referirnos al proceso de enseñanzaaprendizaje (ya sea en modalidades formales, informales, no formales o en procesos de socialización y culturización masivos) prioritarios a los contenidos están los criterios de "aprender a aprender" y "aprender a vivir, ser, hacer, convivir, emprender" para la vida cotidiana personal de todos los miembros de la actual población humana.

Para ser eficaces, los procesos educativos tienen que ser en primer lugar coherentes y pertinentes con el hic et nunc, el aquí y el ahora. Lo que es pertinente en la "educación de adultos" para una mujer jubilada de Suecia, no lo es necesariamente para una mujer campesina, analfabeta de los países pobres, pequeños $\mathrm{y}$ oprimidos. Intencionalmente evito utilizar la expresión "países del tercer mundo" en un mundo en el que ya no existe el antes llamado "segundo mundo".

Si se pasara por el tamiz de la coherencia, pertinencia y eficacia los pensum y programas educativos actuales, veríamos con claridad lo aberrante que resulta la priorización del aprendizaje de la raíz cúbica o de los ríos de Europa, en poblaciones que no saben distinguir una vacuna de un antibiótico, un virus de una bacteria, una gripe de un resfriado y que desconocen la lógica de los interese de un préstamo bancario o los procesos para el registro del nacimiento de un hijo, aún tras haber cursado estudios de educación superior.

Pulula el mercado altamente rentable de cursos importados para formar en el liderazgo, como una panacea para un supuesto éxito en la

14 Cfr. Ismael Cala: "Todos podemos ser emprendedores y líderes" Nicaragua. elnuevodiario.com.ni. 9 de junio de 2013

15 El periodista Cala, también señaló, en sus intervenciones, el tríptico talento, vocación y profesión: "Para emprender algo hay que reconocer cuál es nuestro talento original y qué potenciar para convertirlo en una vocación que será nuestra profesión".

Cala expuso lo que él llama los siete principios para el éxito:1.La calidad de tu vida depende de cómo te comunicas 2.Construye un camino con propósito, no te aferres al destino 3. Cultiva el valor del silencio y la meditación 4. Ríete de la vida, hay que desconfiar del que no tiene sentido del humor 5. Enfoca tu mente en la gratitud: sé feliz con lo que tienes y no pienses en lo que te hace falta 6 . Crea tu propia filosofía y escríbela 7. No negocies tu paz. El autor. 
vida, sin ninguna discriminación de la diversidad de condiciones, planes de vida, edades etc. ¿Qué tienen en común la formación para el liderazgo de un político exitoso, de un militar, de una monja de clausura, de un campesino analfabeta, de un adolescente, de un creyente cristiano o musulmán o budista?

Si realizamos un serio análisis de contenido de estos cursos tan en boga, descubriremos el vacío de respuesta ante preguntas como: ¿existe un modelo universal de liderazgo y cuál es?, ¿un liderazgo por qué, para qué y en función de qué intereses? ¿Si todos incorporáramos el modelo de liderazgo que estos cursos proponen cuáles serían los retos de un mundo en el que todos fuéramos líderes?

Muy pronto descubriríamos que esas propuestas están definidas a priori por la sociedad de mercado que ni quiere ni puede cuestionar el monopolio del poder y del liderazgo del 1\% de la humanidad encuevado en los grandes consorcios económicos y políticos del mundo de hoy.

\section{¿Qué hacer?}

La historia es sabia, si sabemos leerla con honestidad y sentido común. Lo primero, ante el reto evidente de cambio de paradigmas de la humanidad, es tener conciencia, estar convencidos de que los viejos paradigmas que nos rigen, no nos conducen al bien vivir, a una convivencia pacífica -no a pesar sino a partir de las diversidades-, a la imprescindible solidaridad entre las sociedades y entre los individuos.

Esa fue, precisamente la experiencia y el nivel de conciencia que tras la pesadilla de la segunda guerra mundial movilizó mentes y voluntades para intentar cambiar de rumbo. El grito de cambio se sintetizó en el "nunca más una degeneración de esa naturaleza"

Los fundadores de Naciones Unidas y de la UNESCO crearon instrumentos que intentaron ser radicalmente reinventores de una nueva sociedad. En el caso de Naciones Unidas, más que en el caso de la UNESCO, el niño nació con "el cordón umbilical enrollado". Desde su nacimiento los principios como la democracia entre las naciones quedaron condicionados al veto o no veto de los grandes poderes de la postguerra, el Consejo de Seguridad, controlado desde entonces por los vencedores de la guerra. Resoluciones reiteradamente aprobadas por los 192 estados miembros de Naciones Unidas han sido vetadas, a veces por un solo miembro del Consejo de Seguridad, ejemplificando el refrán "todos somos iguales pero algunos somos más iguales".

En junio 2009, la Asamblea General de Naciones Unidas, presidida por el nicaragüense Padre Miguel de Escoto Brockman, celebró una cumbre de líderes mundiales en su Sede, para analizar la peor crisis económica que ha vivido el mundo desde la Gran Depresión. El objetivo fue determinar respuestas de emergencia y de largo plazo para mitigar los efectos de la crisis, especialmente en las poblaciones vulnerables, e iniciar un necesario diálogo sobre la transformación de la arquitectura financiera internacional, teniendo en cuenta las necesidades y preocupaciones de todos los Estados Miembros.

Ese foro de alto nivel concluyó que el reto que enfrentaba la humanidad no se limitaba ni era en primer lugar una crisis financiera sino una familia de crisis imbricadas entre sí: crisis alimentaria, crisis de recursos hídricos, crisis energética, crisis ecológica en general, crisis financiera también y sobre todo, la más grave de todas, una crisis del cambio climático, inducido por el comportamiento del actual modelo de desarrollo.

Así mismo se afirmó que en el enfrentamiento ante estos retos era competencia del G192, es decir de todos los estados miembros de Naciones Unidas y no sólo del G8 o el G20. En esa cumbre la sordera de los grandes poderes fue crónica.

Poco tiempo después el G8 se reunió y se erigió como el gerente absoluto de las crisis.

En los últimos tres años, Miguel de Escoto Brockman ha redactado una propuesta, que ya circula como libro en varios idiomas, para la Reinvención de Naciones Unidas, a partir de la comprobación de la ineficacia evidente de esta organización ante los grandes problemas de la humanidad y su incoherencia con su razón de ser constitutiva.

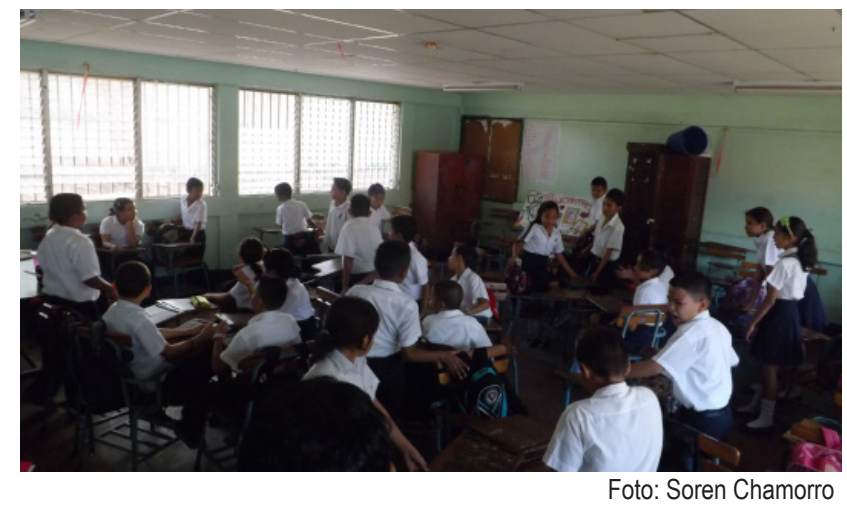


Estas experiencias de la humanidad en las últimas siete décadas, en sus aciertos y en sus fracasos, son ilustradoras para el discernimiento de la adecuada estrategia en pro de la reinvención de los grandes paradigmas.

\section{El camino dialéctico de la metamorfosis}

Amerita señalar que los cambios de los grandes paradigmas de la humanidad están articulados entre sí y todas las nuevas propuestas paradigmáticas luchan por emanciparse de los dogmas suicidas del actual y fracasado modelo socioeconómico, cuya estrategia es convertir toda la realidad, sea ésta material o inmaterial, en mercancía.

Dentro de estos paradigmas que piden desesperadamente una reinvención, más que una reforma, encontramos el paradigma de la educación.

No obstante, la historia nos enseña que este cambio cualitativo no puede realizarse con lógica matemática, sino con lógica dialéctica. Es desde el vientre de la fracasada cosmovisión que rige al mundo actual y no fuera de ella, a través de una sabia administración de las profundas contradicciones del status quo de la sociedad de mercado, que pueden nacer y reinventarse los nuevos paradigmas salvíficos de la humanidad.

Edgard Morin nos dice, recientemente:

"Cuando un sistema no es capaz de resolver sus problemas vitales y fundamentales, ¿qué sucede?

Dos soluciones: este sistema se descompone o entra en regresión y su degeneración se acentúa; o ensaya reformarse pero, cara a la amplitud de transformaciones necesarias, no alcanza a realizar más que una suma de pequeñas reformas.

Cuando un sistema no puede resolver sus problemas fundamentales, puede metamorfosearse, es decir extraer de su seno un metasistema más rico.

Es necesario comprobar que el sistema tierra, hoy en día, no puede resolver ninguno de sus problemas vitales: $n i$ el hambre, ni los problemas económicos, ni las amenazas de guerra nuclear, ni el fanatismo étnico religioso, ni siquiera la dominación del capitalismo especulativo financiero que hace temblar a los estados.

\section{Está condenado a muerte o condenado a la} metamorfosis.

Ésta no puede nacer inmediatamente, ella será el fruto de un proceso histórico.

Si ese proceso histórico es impensable-actualmente, es aún poco probable, continúa siendo posible y, a menudo, lo improbable ha sucedido en la historia-, podemos pensar que las fuerzas salvíficas creadas por la globalización podrán convertirse en fuerzas fecundas.

Podemos pues continuar con esperanza."1617

Llegados este punto de la reflexión surge la pregunta: ¿Cómo, con qué estrategia podemos ir construyendo, en proceso de metamorfosis, el nuevo modelo de educación con calidad salvífica, desde el estatus quo vigente?

Creo que la primera reflexión sobre el qué hacer debe de abordar el desarrollo-educación de la primera infancia dentro de un marco más amplio: la definición autóctona, coherente y eficaz de la Educación Básica.

El reto es grande y muy exigente pero, también es posible, urgente y necesario. Nuestra Nicaragua y nuestra juventud se lo merecen.

16 Morin, Edgard. Pour une gouvernance de la communauté de destins. Le monde n'a plus de temps a perdre.Le Collegium International. Ed. Babel, Paris, 2012. Pag.105 17 Los subrayados y negrillas son del autor.fjl

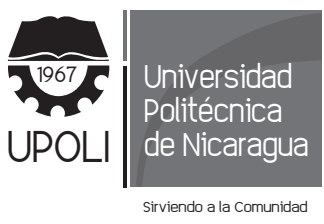

Intituto "Martin Luther King"

Universidad Politécnica de Nicaragua

(UPOLI)

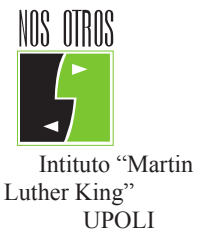

UPOLI 